\title{
Lattice Boltzmann Method for Fast Patient-Specific Simulation of Liver Tumor Ablation from CT Images
}

\author{
Chloé Audigier ${ }^{1,2}$, Tommaso Mansi ${ }^{2}$, Hervé Delingette ${ }^{1}$, Saikiran Rapaka ${ }^{2}$, \\ Viorel Mihalef ${ }^{2}$, Puneet Sharma ${ }^{2}$, Daniel Carnegie ${ }^{4}$, Emad Boctor ${ }^{3}$, \\ Michael Choti ${ }^{4}$, Ali Kamen ${ }^{2}$, Dorin Comaniciu ${ }^{2}$, and Nicholas Ayache ${ }^{1}$ \\ 1 INRIA Sophia-Antipolis, Asclepios Research Group, Sophia-Antipolis, France \\ 2 Siemens Corporation, Corporate Research and Technology, Imaging and Computer \\ Vision, Princeton, NJ, USA \\ 3 Dept. of Radiology, Johns Hopkins Medical Institutions, Baltimore, MD, USA \\ ${ }^{4}$ Dept. of Surgery, Johns Hopkins Medical Institutions, Baltimore, MD, USA
}

\begin{abstract}
Radio-frequency ablation (RFA), the most widely used minimally invasive ablative therapy of liver cancer, is challenged by a lack of patient-specific planning. In particular, the presence of blood vessels and time-varying thermal diffusivity makes the prediction of the extent of the ablated tissue difficult. This may result in incomplete treatments and increased risk of recurrence. We propose a new model of the physical mechanisms involved in RFA of abdominal tumors based on Lattice Boltzmann Method to predict the extent of ablation given the probe location and the biological parameters. Our method relies on patient images, from which level set representations of liver geometry, tumor shape and vessels are extracted. Then a computational model of heat diffusion, cellular necrosis and blood flow through vessels and liver is solved to estimate the extent of ablated tissue. After quantitative verifications against an analytical solution, we apply our framework to 5 patients datasets which include pre- and post-operative CT images, yielding promising correlation between predicted and actual ablation extent (mean point to mesh errors of $8.7 \mathrm{~mm}$ ). Implemented on graphics processing units, our method may enable RFA planning in clinical settings as it leads to near real-time computation: 1 minute of ablation is simulated in 1.14 minutes, which is almost $60 \times$ faster than standard finite element method.
\end{abstract}

\section{Introduction}

In spite of recent advances in cancer therapy, treatment of primary and metastatic tumors of the abdomen, including the liver, remains a significant challenge. Hepatocellular carcinoma (HCC) for example is one of the most common malignancies (more than 1 million cases per year), with increasing frequency in Western countries [1. Unfortunately, less than $25 \%$ of patients with primary or secondary liver cancer are candidates for resection or transplantation, which are considered as the most effective treatments. Consequently, ablative therapies such as radio-frequency ablation ( $\mathrm{RFA}$ ) has raised increasing interest. 
RFA consists in placing a probe within the target area. Electrodes at the tip of the probe create heat, which is conducted into the surrounding tissue, causing coagulative necrosis at temperatures above $50^{\circ} \mathrm{C}$. Success of the procedure depends on the complete coverage of the tumor by the generated necrosis area, which relies on optimal probe placements and the extend of heat delivery. However, the latter is challenged by the hepatic blood vessels that dissipate heat, thus potentially reducing RFA efficiency and increasing risks of recurrence.

Several studies 234] have investigated finite element method (FEM) to compute heat diffusion in liver and predict the optimal placement of the RFA probes. Heat sink as well as various cellular necrosis models [5] have been studied. However, to the best of our knowledge, none of these models rely on patient-specific data. In particular, the vascular system of the liver is neglected or simplified and blood flow circulation is not computed based on patient-specific clinical information. Moreover, FEM is computationally demanding (execution time is in the range of hours), which is not suitable for clinical purposes.

This paper presents a multi-physics model for efficient patient-specific planning of RFA based on medical images such as CT or MRI (Sec. 2). In particular, we rely on the Lattice Boltzmann Method (LBM) to compute heat diffusion in the liver tissue. The LBM offers high scalability, second order accuracy in space and the simplicity of implementation on a uniform Cartesian grid [6]. In Sec. 3 , we demonstrate the validity of our algorithm against an analytical solution and its predictive power is evaluated on patient data. Sec. 4 concludes the paper.

\section{Method}

Fig. 1]illustrates the different steps of our method. Starting from a preoperative clinical CT image, we extract the liver geometry and the venous systems. Next, the bioheat equation is solved using LBM. The liver is highly vascularized and modeling the impact of all vessels is out of reach. Therefore, only the effect of large vessels are described explicitly with the Pennes model [7. Small ones are represented implicitly in the parenchyma as a porous medium using the Wulff and Klinger model [8]. The bioheat equation is weakly coupled to a computational fluid dynamics (CFD) solver to accurately take into account the effect of blood circulation on the dissipated heat, while the blood flow in the porous tissue is computed by solving the Darcy's equation [9]. The heat transfer depends on the blood flow, which is not modified as the organ is heated (the effect of

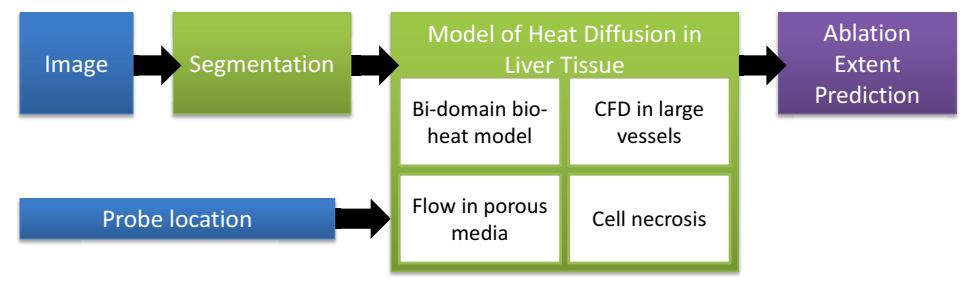

Fig. 1. Steps of the proposed method (blue: input, green: processes, purple: output) 
heat on the viscosity of the flow is neglected as well as the coagulation effect). This assumption allowed us to compute CFD and porous flow only once, at the beginning of the algorithm, thus speeding up the process. LBM and CFD are calculated on a Cartesian grid while the porous solver is executed using FEM on a linear multi-domain tetrahedral mesh for increased accuracy. The resulting flow is tri-linearly rasterized on the Cartesian grid after computation. Finally, a cellular necrosis model is employed to compute cell death due to overheating [5].

\subsection{Model of Patient Liver Anatomy}

Preoperative images are semi-automatically segmented, yielding a detailed anatomical model of patient's liver, including parenchyma, tumors, hepatic veins, vena cava, and portal vein (Fig. 2, left panel). For each structure, we define seeds inside and outside of the area of interest. Then, the random-walker algorithm is employed [10] to automatically estimate the boundaries of the structure. The process can be refined interactively. From the segmentation, a level set representation of the liver, without tumor and vessels is computed. A multi-label mask image is also created to identify the structures of interest for the simulation. Finally, a tetrahedral multi-domain mesh is generated based on the resulting multi-label mesh (www.cgal.org) for computing the porous flow.

\subsection{Model of Heat Transfer in Liver Tissue}

Computing heat diffusion in biological tissues amounts to solving the coupled bioheat equations derived from the theory of porous media, where each elementary volume is assumed to comprise both tissue and blood with a certain fraction. As current imaging techniques do not allow to estimate the accurate ratio between blood and liver tissue, two main simplifications of the bioheat equations have been proposed. The Pennes model [7] assumes constant blood temperature, which holds close to large vessels, where blood velocity is high. The model writes:

$$
(1-\epsilon) \rho_{t} c_{t} \frac{\partial T}{\partial t}=(1-\epsilon) Q+(1-\epsilon) \nabla \cdot\left(d_{t} \nabla T\right)+H\left(T_{b 0}-T\right)
$$

For small vessels, the Wulff-Klinger (WK) model [8] assumes equilibrium between tissue and blood temperatures, with a blood volume fraction $\epsilon \ll 1$ :

$$
(1-\epsilon) \rho_{t} c_{t} \frac{\partial T}{\partial t}=(1-\epsilon) Q+(1-\epsilon) \nabla \cdot\left(d_{t} \nabla T\right)-\epsilon \rho_{b} c_{b} \boldsymbol{v} \cdot \nabla T
$$

In both equations, $T, Q, \boldsymbol{v}$ and $T_{b 0}$ stand for temperature, source term, blood velocity and the mean temperature (assumed constant) of the blood in large vessels. The other parameters are listed in Table 1 In our framework, we use either the Pennes model or the WK model according to the spatial location in the anatomy. Assuming that blood vessels and surrounding tissue are isolated from each other, we compute the temperature by solving the diffusion equation:

$$
\rho_{t} c_{t} \frac{\partial T}{\partial t}=Q+\nabla \cdot\left(d_{t} \nabla T\right)
$$


Table 1. Values from literature [9] of the parameters used in the simulation

\begin{tabular}{|c|c|c|}
\hline parameter & description & value \\
\hline $\begin{array}{c}\rho_{b}, \rho_{t} \\
{ }^{c} b \\
c_{t} \\
{ }_{t}{ }^{*} \\
d_{b}, d_{t} \\
\quad \mathrm{H} \\
\epsilon \\
\kappa \\
\mu \\
\varphi_{v} c_{i n} \\
\varphi_{i} \\
p_{0} \\
k_{f} \\
k_{b} \\
T_{k}\end{array}$ & $\begin{array}{c}\text { blood and tissue densities } \\
\text { blood heat capacity } \\
\text { tissue heat capacity } \\
\text { tissue heat capacity in dead cells } \\
\text { blood and tissue heat conductivities } \\
\text { convective transfer coefficient } \\
\text { blood volume fraction } \\
\text { permeability } \\
\text { dynamic viscosity of the blood } \\
\text { vena cava inflow } \\
\text { flow through the inlets of the hepatic veins } \\
\text { vena cava outlet pressure } \\
\text { forward rate constant } \\
\text { backward rate constant } \\
\text { parameter of cell state model }\end{array}$ & 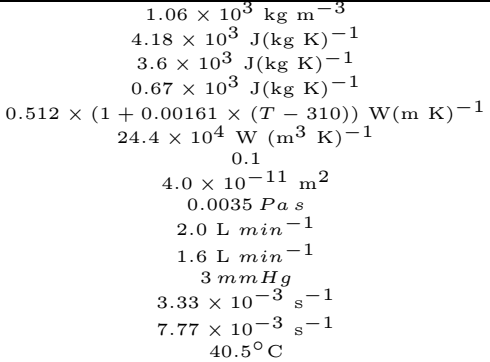 \\
\hline
\end{tabular}

everywhere in the domain, to which we add the cooling term $H\left(T_{b 0}-T\right) /(1-\epsilon)$ when a point belongs to a large vessel (Pennes model) or $-\epsilon \rho_{b} c_{b} \boldsymbol{v} \cdot \nabla T /(1-\epsilon)$ when it belongs to the parenchyma (WK model).

\subsection{Model of the Patient Hepatic Venous Circulation System}

Blood velocity $\boldsymbol{v}$ inside the parenchyma is calculated according to Darcy's law : $\boldsymbol{v}=-\kappa /\left(\mu \epsilon^{2 / 3}\right) \nabla p$ where $p$ is the pressure. This amounts to solving the Laplace equation : $\nabla \cdot\left(-\kappa /\left(\mu \epsilon^{2 / 3}\right) \nabla p\right)=0$. At the border of the liver, Neumann boundary conditions are employed. Dirichlet boundary conditions are applied at the tip of the portal and hepatic veins, to define the pressure drop between them. As we cannot estimate these pressures in-vivo, we rely on a CFD model of the hepatic venous circulation system (Fig. 2 right panel). We used a full 3D CFD solver (incompressible Navier-Stokes equations with viscous terms, expressed in an Eulerian framework which embeds the domain boundary using a level set representation of the segmented vessels [1] ). The blood is modeled as a Newtonian fluid with pre-specified density $\rho_{b}$ and viscosity $\mu$. Let $\varphi_{p}$ be the portal
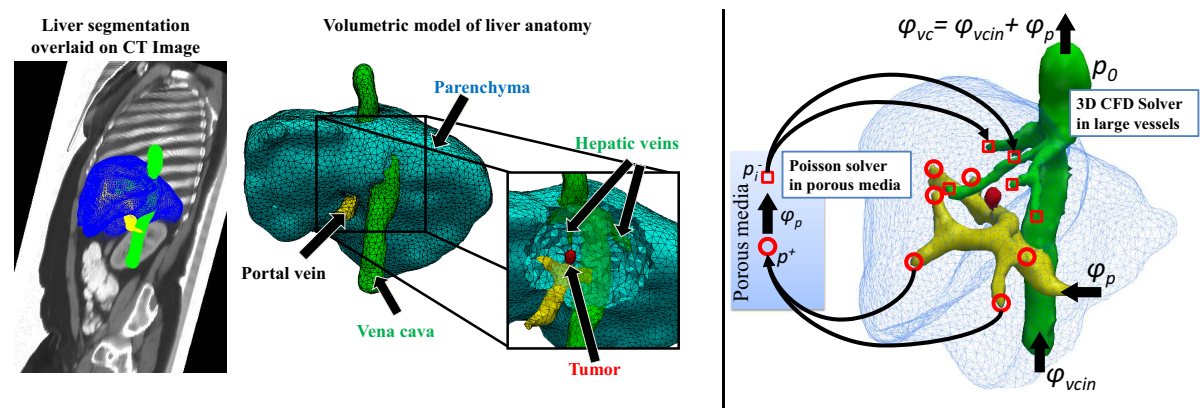

Fig. 2. Left: A detailed anatomical model of the liver is estimated from a standard clinical CT image. Right: Model of the hepatic circulatory system. Arrows denote blood flow. Circles and squares denote portal and hepatic vessel tips. See text for details. 
vein inflow and $\varphi_{v c}=\varphi_{v c_{i n}}+\varphi_{p}$ the vena cava outflow (conservation of mass, the hepatic artery is neglected). We also fix the vena cava outlet pressure in the range of physiological values of healthy patients. The values are listed in Table 1 Blood flow and pressure distribution within the vena cava and hepatic veins: A plug profile velocity field is applied at the inlets (squares in Fig. 2), computed from the outflow $\varphi_{p}$ and each inlet cross-sectional area. The CFD calculation give the downstream pressures $p_{i}^{-}$for each inlet of the hepatic vein. Blood flow and pressure distribution within the portal vein: To estimate the upstream pressure $p^{+}$, assumed constant, of the portal vein outlets (circles in Fig. 2), we solve the Darcy's law and optimize over $p^{+}$such that the computed perfused flow through the hepatic vein inlets matches the one computed using $3 \mathrm{D}$ CFD. Once $p^{+}$is estimated, we compute the blood flow using the CFD solver.

\subsection{Model of Cellular Necrosis}

Tissue necrosis is computed based on the simulated temperatures using a 3state model [5]. The model computes the variation of concentration of alive (A), vulnerable (V) and dead (D) cells over time according to the state equation :

$$
\mathrm{A} \stackrel{k_{f}(T)}{\stackrel{\leftarrow}{k_{b}}} \mathrm{~V} \stackrel{k_{f}(T)}{\longrightarrow} \mathrm{D}
$$

where $k_{f}(T)=\bar{k}_{f} e^{T / T_{k}}(1-A)$ and $k_{b}$ are the rates of cell damage and recovery respectively. This equation results in 3 coupled ODEs solved with a first order explicit scheme at each vertex of the Cartesian grid, yielding a spatially-varying cell state field used in the bioheat solver to update the heat capacity during the computation (Table 1). The initial conditions are chosen as in [5].

\subsection{Lattice Boltzmann Formulation of the Bioheat Equations}

The bioheat model is solved on an isotropic Cartesian grid using LBM with 7connectivity topology and Neumann boundary conditions. For stability reason, we use a Multiple-Relaxation-Time model. The boundaries are treated according to the level set representation using linear interpolation without requiring advanced meshing techniques. The governing equation at position $\mathbf{x}=(x, y, z)$ for the edge $\mathbf{e}_{i}$ is given by (5). $\mathbf{f}(\mathbf{x})=\left\{f_{i}(\mathbf{x})\right\}_{i=1 . .7}$ is the vector of distribution function with $f_{i}(\mathbf{x})$, the probability of finding a particle travelling along the edge $\mathbf{e}_{i}$ of the node $\mathbf{x}$ at a given time, $c=\Delta x / \Delta t, c_{s}^{2}=1 / 4, \Delta x$ is the spacing.

$$
\mathbf{f}\left(\mathbf{x}+\mathbf{e}_{\mathbf{i}} \Delta x, t+\Delta t\right)=\mathbf{f}(\mathbf{x}, t)+\mathbf{A}\left[\mathbf{f}^{e q}(\mathbf{x}, t)-\mathbf{f}(\mathbf{x}, t)\right]+\boldsymbol{\omega} \Delta t H\left(T_{b 0}-T(\mathbf{x}, t)\right)
$$

$f_{i}^{e q}(\mathbf{x}, t)=\omega_{i} T(\mathbf{x}, t)\left[1+\frac{\mathbf{e}_{\mathbf{i}} \cdot \mathbf{v}}{c c_{s}^{2}}\right]$ and $\boldsymbol{\omega}=\left\{\omega_{i}\right\}_{i=1 . .7}$ the vector of weighting factors [6]. The temperature is computed as $T(\mathbf{x}, t)=\sum_{i=1}^{7} f_{i}(\mathbf{x}, t)$ and is updated at every node of the grid for every timestep. Finally, we model the heat source term through a Dirichlet boundary condition at the location of the probe. 


\section{Experiments and Results}

All experiments were executed on a Windows 7 desktop machine (Intel Xeon, 2.80 GHz, 45GB RAM, 24 CPUs) with a Nvidia Quadro 60001.7 GB (448 CUDA cores).

\subsection{Quantitative Verification against Analytical Solution}

To evaluate our model, we compared its behavior on a regular cuboid domain with the $3 \mathrm{D}$ analytical solution of a source of mass $\mathrm{M}$ released at $\mathbf{x}_{\mathbf{0}}$ at time $t_{0}$ :

$$
T(\mathbf{x}, t)=\frac{M}{\left[4 \pi\left(t-t_{0}\right) D\right]^{3 / 2}} \exp \left(-\frac{\left\|\mathbf{x}-\mathbf{x}_{\mathbf{0}}-\left(t-t_{0}\right) \boldsymbol{v}\right\|^{2}}{4 D\left(t-t_{0}\right)}\right)
$$

of the advection-diffusion equation : $\frac{\partial T}{\partial t}+\mathbf{v} \cdot \nabla T=\nabla \cdot(D \nabla T)$. Parameters were chosen to get heat diffusion in physiological range: $D=0.1 \mathrm{~mm}^{2} / \mathrm{s}, \boldsymbol{v}=$ $(2,0,0) \mathrm{mm} / \mathrm{s}, M=35000^{\circ} \mathrm{C} / \mathrm{mm}^{3}, t_{0}=-50 \mathrm{~s}$, yielding a Gaussian-shape source term of $70^{\circ} \mathrm{C}$ at the center at time $t=0$. In our LBM solver, we initialized the temperature values at each point with the analytical solution at time $t=0$. The temperature at several points of the domain was reported. Our implementation was qualitatively close to the analytical solution (Fig. 3). For a given resolution, an upper and lower bound for the time-step were provided by the simulated physics and the Courant-Friedrichs-Lewy conditions. As expected, the smaller the spatial resolution, the more accurate the solution. A time-step of $75 \mathrm{~ms}$ and a resolution of $1-2 \mathrm{~mm}$ appeared to be a good compromise between accuracy and computational cost. From a computational point of view, experiments showed a speed-up of 11 with parallel optimization (OpenMP) and 45 with graphical processing units (GPU) implemented on CUDA with respect to a single-core implementation of LBM. After a quantitative verification of the FEM simulation against the analytical solution, experiments showed that a $60 \times$ speed-up was obtained with respect to FEM for a similar accuracy.
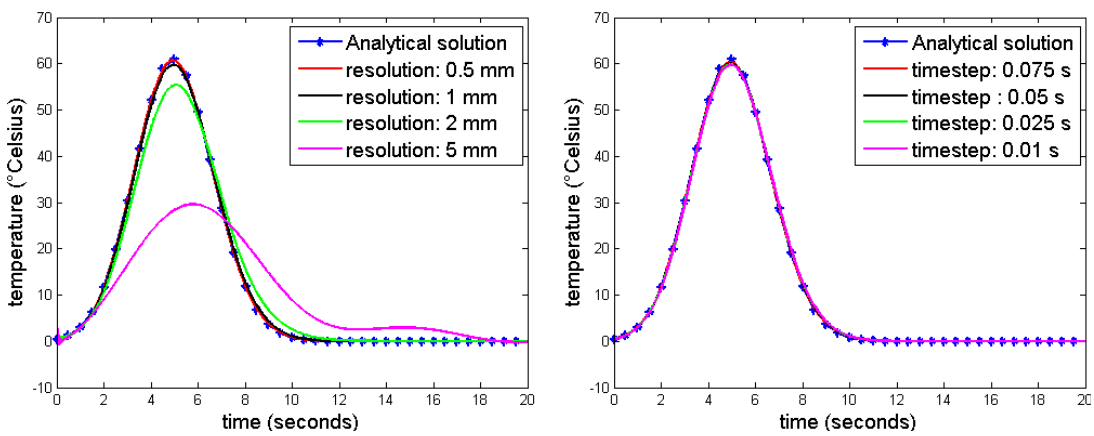

Fig. 3. Left: Spatial convergence analysis for a fixed time-step of $0.01 \mathrm{~s}$. Right: Time convergence analysis for a resolution of $1 \mathrm{~mm}$ compared to the analytical solution. As one can see, the proposed framework quickly converges to the right solution. 


\subsection{Evaluation on Patient Data}

We evaluated our model on 5 patients, with 7 ablations (patient 5 had 3 tumors ablated) for whom pre- and post-operative CT images were available. For all patients, nominal tissue parameters were employed. Clinical RFA protocol requires that the probe is deployed within the tumor with a diameter defined pre-operatively according to the size of the tumor, and then maintained for 7 minutes after the target temperature of $105^{\circ} \mathrm{C}$ was reached, as measured by the probe thermisters. For large tumors, the process was iterated with sequentially increasing diameters. After anatomical model extraction, we emulated the RFA protocol by placing the virtual probe at the center of the tumor. Cells around the probe tip within the probe diameter sphere were heated at $105^{\circ} \mathrm{C}$ during 7 minutes or 2 times 7 minutes. The simulation continued for 3 more minutes without the probe so that each cell reach a steady state. Qualitatively, computed ablation followed closely the boundaries of the vessels, due to the heat sink effects of the blood. The shape of the ablated area also depended on the heat advection due to the small arteries (Fig. (4). Cell death area computed using the model compared qualitatively well with the observed postoperative necrosis zone (Fig. 4, the lesion was manually segmented by an expert and rigidly registered to preoperative image). Quantitatively, average point-to-mesh errors (Table 21) were within clinical variability as they were lower than the different size configurations of the probes. More importantly, the simulation predicted that the selected protocols covered the entire lesion, which is the clinical criterion for ablation planning. One minute is computed in 1.14 minutes, in comparison, using FEM it takes 1.14 hours. To the best of our knowledge, this is the first time that near realtime simulations of RFA ablation could be achieved. All cases presented a larger necrosis area compared to the ground truth, the diffusion coefficient used from the literature was too high to get a perfect match.

\section{Discussion and Conclusion}

We have presented a first patient-specific model of liver tumor ablation allowing near real-time computation. As we rely on LBM, our framework does not require advanced meshing techniques to solve bioheat equations and the level set representations of the structures are directly computed from images. We focused on

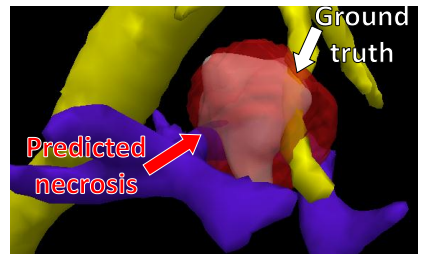

Fig. 4. Predicted necrosis compared qualitatively well with ground truth (patient 2)
Table 2. Evaluation on Patient Data

\begin{tabular}{|c|l|l|}
\hline patient & point-to-mesh error & probe diameter \\
\hline 1 & $9.5 \pm 5.9 \mathrm{~mm}$ & $4 \mathrm{~cm}$ then $5 \mathrm{~cm}$ \\
2 & $4.6 \pm 3.3 \mathrm{~mm}$ & $3 \mathrm{~cm}$ \\
3 & $10.4 \pm 6.5 \mathrm{~mm}$ & $4 \mathrm{~cm}$ then $5 \mathrm{~cm}$ \\
4 & $11.7 \pm 6.3 \mathrm{~mm}$ & $4 \mathrm{~cm}$ then $5 \mathrm{~cm}$ \\
$5-1$ & $7.3 \pm 5.1 \mathrm{~mm}$ & $4 \mathrm{~cm}$ then $5 \mathrm{~cm}$ \\
$5-2$ & $8.2 \pm 6.2 \mathrm{~mm}$ & $4 \mathrm{~cm}$ then $5 \mathrm{~cm}$ \\
$5-3$ & $9.0 \pm 5.5 \mathrm{~mm}$ & $4 \mathrm{~cm}$ then $5 \mathrm{~cm}$ \\
\hline
\end{tabular}


modeling heat propagation and cell death based on a patient image taking into account the heat sink effect of blood vessels and porous circulation in the liver. Despite possible biases in establishing correspondences between the post- to the pre-operative images, and the use of nominal biological parameters, which are not patient-specific, our model provided promising results, opening new opportunities in the RFA planning and guidance, even if the target is to go beyond real-time, as we need 1-2 minutes of computation for clinical use. We have not considered the effects of the arterial flow, but it would be straightforward to include it for improved accuracy, provided the hepatic artery is visible in the image. It is worth noting that the veins account for more than $70 \%$ of the blood flow 12. Future works include validation on larger cohorts of patient, and a full coupling of blood flow and heat transfer models for more accurate predictions.

Acknowledgments. We thank Marta Mesa Gonzalez for segmenting all the cases.

\section{References}

1. El-Serag, H.B., Davila, J.A., Petersen, N.J., McGlynn, K.A.: The continuing increase in the incidence of hepatocellular carcinoma in the united states: An update. Ann. Intern. Med. 139, 817-823 (2003)

2. Chen, X., Saidel, G.M.: Mathematical modeling of thermal ablation in tissue surrounding a large vessel. J. Biomech. 131 (2009)

3. Jiang, Y., Mulier, S., Chong, W., Diel Rambo, M., Chen, F., Marchal, G., Ni, Y.: Formulation of 3D finite elements for hepatic radiofrequency ablation. IJMIC 9, 225-235 (2010)

4. Kröger, T., Pätz, T., Altrogge, I., Schenk, A., Lehmann, K., Frericks, B., Ritz, J., Peitgen, H., Preusser, T.: Fast estimation of the vascular cooling in RFA based on numerical simulation. Open Biomed. Eng. J. 4, 16-26 (2010)

5. O’Neill, D., Peng, T., Stiegler, P., Mayrhauser, U., Koestenbauer, S., Tscheliessnigg, K., Payne, S.: A three-state mathematical model of hyperthermic cell death. Ann. Biomed. Eng. 39, 570-579 (2011)

6. Rapaka, S., Mansi, T., Georgescu, B., Pop, M., Wright, G.A., Kamen, A., Comaniciu, D.: LBM-EP: Lattice-boltzmann method for fast cardiac electrophysiology simulation from 3D images. In: Ayache, N., Delingette, H., Golland, P., Mori, K. (eds.) MICCAI 2012, Part II. LNCS, vol. 7511, pp. 33-40. Springer, Heidelberg (2012)

7. Pennes, H.H.: Analysis of tissue and arterial blood temperatures in the resting human forearm. J. Appl. Physiol. 85, 5-34 (1998)

8. Klinger, H.: Heat transfer in perfused biological tissue I: General theory. B. Math. Biol. 36, 403-415 (1974)

9. Payne, S., Flanagan, R., Pollari, M., Alhonnoro, T., Bost, C., O’Neill, D., Peng, T., Stiegler, P.: Image-based multi-scale modelling and validation of radio-frequency ablation in liver tumours. Philos. T. Roy. Soc. A. 369, 4233-4254 (2011)

10. Grady, L.: Random walks for image segmentation. IEEE T. Pattern Anal. Mach. Intell. 28, 1768-1783 (2006)

11. Ralovich, K., et al.: Hemodynamic assessment of pre- and post-operative aortic coarctation from MRI. In: Ayache, N., Delingette, H., Golland, P., Mori, K. (eds.) MICCAI 2012, Part II. LNCS, vol. 7511, pp. 486-493. Springer, Heidelberg (2012)

12. Schenk Jr., W.G., McDonald, J.C., McDonald, K., Drapanas, T.: Direct measurement of hepatic blood flow in surgical patients: with related observations on hepatic flow dynamics in experimental animals. Ann. Surg. 156, 463-469 (1962) 